to such cases as these I found two years ago in the dissecting-room, when the urethra was deficient in its upper surface, the glans was completely bifid, and a deep groove led along its medial line to the corona glandis, where the urethra commenced as in the other cases, but upon its upper surface. I shall have occasion in my next lecture to refer to a remarkable case of the ordinary congenital deficiency of the urethra, in speaking of the specific distance of gonor-. rhœa. In congenital phymosis the prepuce retains its natural structure, but its opening is so small that the patient can only denude the orifice of the urethra and a very small portion of the glans. The glans, as well as the penis itself, is well developed. There is a patient at present in attendance with this affection; his name is Abbott, and his age is 22 years. The size of the orifice of his prepuce is nearly that of a sixpence, and although he has had gonorrhœa three times, there is no alteration in the structure of the part. It is of importance to distinguish in our treatment these cases of phymosis from those which occur from cicatrices or thickening of the prepuce, as different operations are applicable to each; indeed, the latter kind of phymosis is often removed by the cautious administration of mercury.

Hernia humoralis, or gonorrhœal testitis more properly called, in most cases arises from neglect and irregularities, on the part of the patient. We have had 107 cases of this disease, which to the cases of gonorrhœa bears the proportion of 1 in 20 . This, however, is not a fair criterion to go by, for certainly more than one of the patients affected with this kind of testitis did not apply to us until the disease was fully set in. It is a rare occurrence to find testitis supervening if the patient is judiciously treated for gonorrhœa, and he observes strictly the injunctions laid upon him. I shall spcak of the other forms of testitis we have met with when hernia humoralis comes to be specially considered.

Hydrocele of the tunica vaginalis and of the spermatic chord occurs from a very early period of life to a very late one. In the adult both diseases require much more active treatment than in early life; a mere puncture in the latter will be sufficient in most instances to cure one or the other, whereas in the adult we have had occasion here to use not unly a variety of injections but also the seton.

The number of secondary syphilitic cases, which have been admitted to this institution, bear to the number of cases affected with primary symptoms a propartion of something more than one to three. For the same reason $I$ gave in speaking of gonorrhœal testitis, no sound conclusion can be drawn from these premises. It is a most unusual occurrence to find the patients who are treated here for primary syphilis, applying again with secondary symptoms. The cases of secondary syphilis which do apply have in general been under treatment somewhere elsc, and are truly primary patients with us. This important disease I shall particularly direct your attention to in future lectures. In regard to the patients admitted since we last met, and to which I alluded at the commencement of this lecture, time will only permit me to direct your attention to the cases of Gell, Connor, and Field! in each of whom I opened the dorsal vein of the penis for gonorrhœa in its most aggravated form.

\section{PRACTICAL OBSERVATIONS}

ON

\section{I S A E S OF THE SKIN.}

By Thomas H. Burgess, M.D., \&c.

No. I.

ERUPTIONS OF THE FACE.

No branch of pathology abounds with greater in. terest or opens a more fertile field for pathological research than that which embraces a consideration of the nature, causes, and treatment, of diseases of the skin; yet, strange to say, no other department of medicine has been less cultivated in this country of late years ; and whilc medical science, generally speaking, has advanced, pari passu, with the enlightened spirit of the age, this branch of it has been allowed to remain almost in the same state as when Willan and Bateman wrote. Fortunately, however, so important a subject has not been equally neglected in other countries, and to France especially is due the merit of having zealously followed out the principles correctly laid down by the distinguished writers above mentioned. Thus we find the names of Alibert, Biett, Rayer, Cazenave, and others, who have at various periods been attached to the Hospital of St. Louis, associated with all those researches and improved views in dermatology which have issued during the last twenty years from that admirable institution.

It is pretty generally admitted that one of the principal sources of the error which has so long prevailed in the history of skin affections is owing to the want of opportunity of observing the various eruptions from their commencement to their termination -a contingency which must always occur while the practitioner has to depend on the irregular attendance of out-patients for his information. This class of patients, it is needless to say, only attend the hospital or dispensary when it suits their convenience and inclination to do so, especially when no urgent symptoms are present, and in nine cases out of ten the phy sician is enabled to see the disease but twice or three times during its progress. When we recollect the slight difference that exists betwcen a vesicle and a pustule in certain stages of their course, that the bulla of rupia often closely resembles the pustule of ecthyma, and moreover, that the fluid of a vesicle may dry into a scab or incrustation, the fallacy of such a means of arriving at pathological facts and precise descriptions of disease must be sufficiently obvious. To understand thoroughly the nature of the important class of affections under consideration, it is necessary that they should pass before the eye of the student in every phase of their existence, and this desideratum is only to be obtained in an institution like the Hospital of St. Louis, where the patients are admitted and retained during the progress of the disease. I propose in this, and a few subsequent papers, to describe briefly those cutaneous eruptions which are of the most frequent occurrence, adopting for my guide the plan pursued by $\mathbf{M}$. Biett and $\mathbf{M}$. Cazenave at the Hospital of St. Louis during my attendance at that institution.

Of all the eruptions to which the skin is liable, and they are numerous and variable, none excite greater 
anxiety in the mind of the paticnt or practitioner than those which occur on parts of the body constantly exposed to view, as, for example, the hands, the neck, the face, the scalp, \&c. It is unnecessary to dwell here on the importance of accurate acquaintance in a diagnostic point of view with the particular affections alluded to; for although they may not be sufficiently serious to involve life, still their existence is a source of continual annoyance to the patient, and nothing is more likely to prolong the evil than an error in diagnosis at the onset.

Eruptions of the face are so numerous, and above all so conspicuous, that they demand our attention in the first place. The tendency of the capillary system of the face to be overrun with blood, disposes it to become more frequently the seat of a multitude of affections than any other part of the body. Erysipelas, for example, is more frequently detected in this region than in any other; it is the principal seat of the pustules in small-pox, and of other equally familiar examples. The face may be attacked by almost every disease to which the skin is liable; but I only intend to treat in this place of those particular affections which appear most frequently in that region. The mildest affection, perhaps, that occurs on the face is that variety of pityriasis, which the French call dartre farineuse. It is met with in individuals of a delicate transparent skin, and especially in young girls. It is characterised by a peculiar mealy or farinaceous desquamation, which distinguishes it from every other affection, and is so mild and transitory in its nature as to require scarcely any treatment. If it should persist, however, longer than usual, a mild laxative and any bitter infusion containing from one to two drachms of the carbonate of potass to the pint will soon effect a cure.

The same class of individuals are also subject to another mild eruption of the face-herpes circinatus -which bears some resemblance to the preceding affection in its farinaceous appearance; but the desqua mation is not so abundant in this as in the former case. This eruption is frequently seen in the form of rings on the cheeks and chin of young people of either sex of a fair and delicate skin. The rings are studded round with extremely small globular vesicles. Their centre is generally free, and the border red. When the herpetic rings are small the circular border is pretty broad, and the red color extends beyond the vesicles to the same distance on either side. The vesicles usually terminate about the eighth or tenth day by desquamation, leaving merely a slight degree of redness behind, which gradually disappears. The rings of herpes circinatus are liable to be mistaken for diseases of a much more serious nature and very different character to itself-namely, lepra, porrigo scutulata, lichen circumscriptus, and I may take this opportunity of observing that one of the most important features connected with the history of cutaneous diseases generally, and those of the face especially, is their differential diagnosis. Amongst the great variety of eruptions to which the face is subject, it is obvious that there must be a considerable variation in the degree of severity of each, and in their appropriate treatment; hence the importance of an accurate knowledge of their diagnosis.

For example, a small herpetic ring, the vesicles of which are slightly exfoliated, situated on a perfectly round and red surface, may often be mistaken for a patch of lepra without scales. It is needless to dwell on the evil results, both as regards the treatment and the peace of mind of the patient, sure to follow an error of the kind; but happily this mistake may easily be avoided, and cannot occur to any careful observer, for the depression in the centre and the prominent border of the leprous patch, may readily be distinguished from the even surface and the debris of vesicles so characteristic of the rings of herpes. Besides, there are generally several patches of lepra present at the same time, some of which are sufficiently developed to indicate their real nature. Again, herpes circinatus may be confounded with porrigo scutulata, the name ringworm being erroneously applied to both. However, one is a vesicular and the other a pustular affection. The latter is of long duration, forms thick scabs, is contagious, destroys the hair when it occurs in that neighbourhood, while the former is a mild affection, lasts but a short time, is not contagious, never destroys the hair, and terminates by desquamation, or by insensible exfoliation. The rings of lichen circumscriptus are not much larger than those of herpes circinatus, and hence it is sometimes difficult to distinguish one from the other. Here we must depend principally on the nature of the elementary lesion for a means of diagnosis, which in the former case is a papule, whilst in the latter it is a vesicle. The nature of the eruption once ascertained, the treatment is exceedingly simple, and very similar to that required for the variety of pityriasis already described, the only additional remedies necessary being mild alkaline lotions. The disease is very slight, and is not long in disappearing.

There is another variety of herpes-herpes labialis frequently met with on the face. It is generally produced by cold air, by the contact of acrid fluids, or by gastric derangement. Although, generally speaking, it is a mild affection, it is, nevertheless, frequently ushered in by an exceedingly smart feverish attack. This variety appears in the form of small groups of vesicles scattered irregularly round the lips; some of these vesicles remain distinct, whilst others run into one another and form a small scab. The surface on which the eruption is developed becomes red and swollen, and it is attended by a disagreeable smarting sensation. The vesicles are of various sizes, but the largest does not exceed the size of a small pea. Herpes labialis runs its course rapidly. The incrustations formed by the debris of the vesicles disappear in the course of three or four days, leaving a small red stain behind, which in its turn gradually fades off. If not injudiciously interfered with, this slight affection requires scarcely any treatment; a mild aperient, and a lotion containing two or three grains of sulphate of zinc to the ounce are lall the remedial measures necessary. The progress of the eruption is sometimes shortened by transfixing the vesicles individually as they ripen with a fine-pointed needle, and allowing the contained fluid to escape without exposing the surface.

Lichen.-There is another affection, of a severer character than either of the foregoing, which we fre quently observe on the face in young persons-that is lichen agrius. It is characterised by an eruption of very small accuminated, inflamed papulæ, developed 
on an erythematous surface, and accompanied by severe heat and smarting, instead of the distressing pruritus which attends the other varieties of lichen. The papulæ become confluent, and are surrounded by a small reddish areola. They soon become red themselves. Slight ulcerations form on their apices, whence issues a sero-purulent fluid, which concretes, and forms yellowish incrustations of various degrees of density. The disease may continue in this state for several weeks, or under peculiar circumstances it may pass into the chronic state and terminate by slight exfoliation. It does not interfere with the general health of the patient, and generally attacks those of strong and vigorous constitutions. Although the diagnosis of lichen is often attended with much difficulty, this variety of the disease can hardly be mistaken for any other eruption of the face. Even in its most confluent form there are always papulæ to be found scattered round the morbid parts, which will readily distinguish it from impetigo, eczema, and psoriasis-the only diseases to which it bears any resemblance. When the eruption assumes an obstinate and severe form, strict regimen and a course of mild purgatives will be necessary; sometimes the application of leeches round the diseased parts is attended with considerable benefit at the onset, and at a later period dilute nitric or sulphuric acid administered in barley water will be found very serviceable. In extreme cases the arsenical preparations have been found necessary, but I shall reserve my remarks on this point for a future period, when I purpose treating of lichen generally.

Impetigo.-A variety of this disease, known under the name of impetigo figurata, appears more frequently on the face than on any other part, and gives the countenance a peculiar and repulsive appearance. It generally attacks the same class of persons as the two previous eruptions, and is liable to occur periodically for years. It first appears in the form of small, distinct, red, and slightly raised patches, which soon become studded over with minute pustules, finally running into one another. These patches generally remain distinct, but they sometimes coalesce by the development of a fresh crop of pustules between them, and then the disease spreads considerably over the face. At certain irregular periods a variety of examples of this disease are to be seen in the wards of the hospital of St. Louis, while at others there is scarcely a single case present. The influence of season may have something to do with this epidemic character of the disease, if I may so call it, for I have observed that more cases of the eruption occurred in spring than at any other period during my attendance at that hospital. But in other years that season passed over without the appearance of the disease. In some instances $I$ have seen the eruption commence on the wings of the nose, and spread gradually to the cheeks on either side; and in other cases it only appeared on one cheek or on the chin, where it remained, and pursued the usual course. The pustules burst in the course of thirty-six to forty-eight hours, when the contained fluid concretes and forms scabs, which increase in volume by a continued exudation from the diseased surface. These incrustations present a yellowish appearance in some points and are greenish in others, and are not unlike layers of concrete honey or the gummy exudation of certain trees. The disease sometimes appears on the lips, in the form of moustaches, when the scabs are thick and the subjacent parts indurated and swollen. The duration of this eruption is very variable. In some cases a cure is accomplished in the course of fifteen days, whilst in others the eruption persists for a much longer period, especially when successive eruptions appear. High living, violent exercise, and strong moral passions are frequent causes of the disease.

Impetigo figurata can hardly be confounded with other diseases of the face by any careful observer, yet instances have occurred in which the scabs that appear on that region during the syphilitic eruption have been mistaken for those of impetigo. It is needless to say that such an error could not occur to any person at all acquainted with the characteristic phenomena of either complaint. When the disease under consideration appears on the chin, it requires some little attention to distinguish it from sycosis, another eruption of the face which we shall have to speak of by and by. The pustules of impetigo are small, yellow, and set close together, the scabs are thick and of a yellowish green color, whilst those of sycosis are much larger, not confluent, and not so yellow as the former. Besides the exudation is by no means so copious in sycosis as in impetigo figurata, and the scabs are of a darker color.

When impetigo figurata assumes an acute character, it is, generally speaking, a slight affection, and requires but simple treatment. When the inflammatory symptoms are pretty smart, a few leeches should be applied behind the ears ; but in general cooling lotions, acidulated drinks, and a few mild laxatives, are the only measures required, at least at the commencement of the treatment. At a later period weak alkaline lotions and the vapor douche may be employed with benefit. The preparations of sulphur should not be used in the acute form of this eruption. They often do a great deal of mischief, and are only to be administered when it assumes a chronic character, and even then with caution.

Psoriasis.-There are two varieties of this disease which may be classed amongst the eruptions of the face-these are, psoriasis ophthalmica and psoriasis labialis. These eruptions invariably appear in the form of a circle of three-quarters of an inch or one inch in breadth, which completely surrounds the mouth or the eyelids, as the case may be. This circle, which is composed of pretty large scales, is indented with a number of transverse lines, which extend to the borders of the lips or eyelids, giving to these particular parts an extremely disagreeable and unseemly appearance. The eyelids are often much swollen, and become tender and painful when moved. When the disease is confined to this part it is frequently attended by a smart itching, and sometimes spreads to the conjunctiva, when it assumes a very obstinate character. Psoriasis labialis should not be confounded with eczema, which it often closely resembles. Both diseases exhibit the same kind of chaps or fissures; but the absence of vesicles - the elementary lesion of the latter - the large size and hardness of the scales, and the thickness of the epithelium, are sufficiently diagnostic of the former to prevent a mistake of this kind from occurring. These eruptions of the face are, generally 
speaking, very obstinate and very rebellious. They require the same plan of treatment as psoriasis in general, which I may have occasion to speak of at a future period, and shall therefore dismiss the subject for the present, merely observing that the application of three or four leeches behind each ear at the commencement of the treatment in psoriasis ophthalmica, and at a later period frictions, with an ointment composed of the protochloruret of mercury, which is also an appropriate remedy in cases of psoriasis of the lips, will be found exceedingly beneficial.

Before concluding, I would beg to call the reader's attention again to the importance of accuracy of diagnosis in cutaneous pathology, and the necessity of this may be seen from the striking difference-a difference not only of degree but of kind-which existed in the nature and severity of the various eruptions of the face described in this paper. For example, we have seen that the two first-pityriasis and herpesare very slight and transitory affections, if they are not injudiciously interfered with, or mistaken for other eruptions requiring more active treatment. How different from these in their nature and progress are the varieties of psoriasis which attack the lips and eyelids! We have seen that the latter are often rebellious and intractable diseases, and yet the regions they occupy are not unfrequently the seat of other eruptions of a different order and far milder character, and, nevertheless, one has been mistaken for the other. In a word, the treatment of a great number of cutaneous affections is but of secondary importance compared with their differential diagnosis. Many of them will get well almost without any treatment, provided they are allowed to pursue their natural course; and, on the other hand, a mild and simple eruption, by being mistaken from a similarity of external appcarances for one of a severe and rebellious character, and treated accordingly, may be aggravated and prolonged for an indefinite period. A case in point came under my observation at the Hospital of St. Louis in 1836, in which herpes labialis had been mistaken for psoriasis of the lips, and sent to the hospital under that impression. However anomalous such an error may appear, the parts were so completely altered from the appearance they usually present when the seat of the vesicular eruption, by the erroneous treatment employed before admission, that it required considerable care and attention to ascertain the nature of the disease. There was no elementary lesion present, and the diseased, surface was abraded, chapped, and furrowed. The appearance of a few vesicles the following day, however, revealed its true nature, and a cure soon followed. The patient was suffering at the same time from eczema of the legs.

29, Margaret-street, Cavendish-square. Dec. 30, 1842.

SUDDEN DEATHS AT STRASBOURG.

The causes of death, as ascertained in twenty-six cases of sudden death at Strasbourg, by post-mortem examination, were as follows:-Apoplexy (cerebral hæmorrhage), 1 ; scrous apoplexy, 1 ; cerebral congestion, 4 ; cerebral and pulmonary congestion, 1 ; hæmoptysis, 1 ; foreign bodies in the bronchi, 2 ; pulmonary congestion, 13 ; syncope, 1 ; perforation of the intestiues, 2.-Total, 26.

\section{ON THE USE}

OF THE

\section{PROTO-IODURET OF IRON \\ IN THE}

TREATMENT OF PHTHISIS.

By A. Dupasquier,

Physician to the Hotel-Dieu of Lyons.

After having devoted five or six years to experiments with the ioduret of iron in cases of phthisis, I published, about a year ago, some observations on the mode of administering this remedy. It had been my intention to follow up this memoir by one on the treatment of pulmonary consumption with the proto-ioduret of iron, but as I have hitherto been prevented from want of time, I avail myself of an interesting paper on the same subject, drawn up by M. Boissière, one of my internes. This paper I publish without the slightest alteration, merely adding to it a few notes.

On the Use of the Proto-ioduret of Iron in Phthisis By M. Gilbert Boissière, late interne at the HotelDieu, Lyons.?

The present memoir is founded on the study of twenty-seven cases of pulmonary consumption, observed during a period of four months, in the wards of M. Dupasquier. The restricted number of patients, and the short time during which they were submitted to observation, must plead as an excuse for the imperfections of my essay; still upon some points I have been able to collect a number of facts sufficient for the deduction of certain conclusions.

I must observe, in the outset, that it was much easier to determine the influence of the proto-ioduret of iron on certain functions and on isolated symptoms, than its action on the progress of the disease, taken as a whole; to ascertain the former a few days' observation sufficed, to settle the latter it would have been necessary to follow every case to its termination, but of the twenty-seven cases which I observed, seven died before the remedy had time to act, and of the remainder only one was discharged cured. Still, though we can reckon only a single cure, the improved condition of the other patients was sufficiently striking to authorise me to declare that the proto-ioduret of iron is the most efficacious remedy that the physician can have recourse to in cases of pulmonary consumption.

Physiological Action.-Under this head I would place all the phenomena observed during the use of the remedy, and which have no relation to the symptoms of phthisis. They are primary or secondary.

a. Primary Effects.-Having experienced these myself, after taking a dose of twenty drops, * and having found, on questioning the patients, that they experienced similar effects, it will suffice to relate what I felt myself. Before taking the proto-ioduret, I examined the pulse carefully on three occasions, at intervals of ten minutes, and found that it beat regularly seventy-five times in the minute. After a quarter of an hour the pulse had risen to 85, and I felt some

- The preparation employed by M. Dupasquier is a pure proto-ioduret of iron, in which the iodine is completely saturated by the iron. The solution, which is very similar to that prepared by $\mathrm{Mr}$. Squire, contains one part of the salt to five of water.-EDs. 